\title{
Scents and scentsitivity - what scents (may) spell out and ways to read it
}

\author{
Carsten T Muller*, Eleanor F Kean, Elisabeth A Chadwick, Natasha D Spadafora, Hilary J Rogers \\ From 1st International Workshop on Odor Spaces \\ Hannover, Germany. 4-7 September 2013
}

Chemically, scent marks and natural flavours mostly consist of complex mixtures of chemical compounds, which are neither easily recorded nor interpreted. The facts that the size of a signal in the chemical detection (mass spectrometer) does not necessarily relate to the size in perceptual space and that a signal can just as well be encoded as bouquet of compounds than depending on presence and absence of multiple or single components complicate matters even further. In a recent project on European otters and an ongoing EU project on fruit and vegetable flavours we addressed these issues by using novel approaches in data evaluation and sample collection and data acquisition.

In both projects the essential first steps in data processing involved construction of retention-indexed custom mass spectral libraries, which were subsequently used for deconvolution, putative identification and integration of GC-MS data. Retention indexing proved to be essential for reliable re-identification of components between samples and for putative identification of chemicals from NIST library. In the case of European otters, we had access to a large inventory (>600) of scent glands collected by the Cardiff University Otter Project during post mortem examination of otters found dead in England and Wales over the past decade, which were complemented by a wealth of information on e.g. sex, age, developmental stage, health.

More traditional multivariate methods, e.g. discriminate function analysis enabled us to discriminate sex, age and developmental stages after SPME sampling of headspace, GC-MS analysis and data processing. For subsequent evaluation involving genetic data sets we did change the statistical methods using approaches developed for ecological datasets, such as PerMANOVA, which allow direct comparison of bouquets.
This general approach is currently followed and refined in the EC project QUAFETY, in which we aim to identify markers for quality and safety of ready-to-eat fresh-cut fruit and vegetable. Because of the perishable nature of the odour sources (melon and rocket) we decided to sample headspace on thermodesorption (TD) tubes and devised a simple, easy to use and robust sampling protocol, which was successfully used by our consortium partner organizations across Europe to sample headspace alongside their own experiments. In addition to the use of TD analyses were carried out on GC- time-of-flightMS, which increased sensitivity on average by a factor of 50. The analytical set-up and application of PerMANOVA and canonical analysis of principle ordinates for evaluation of the data allowed successful discrimination of complete scent profiles from $100 \mathrm{~mL}$ of headspace of melon samples according to age and cut size. Currently we are in the process of applying further statistical methods, e.g. weighted correlation network analysis with the aim to identify subsets of compounds, which correlated with single traits and conditions.

Published: 16 April 2014

doi:10.1186/2044-7248-3-S1-P11

Cite this article as: Muller et al: Scents and scentsitivity - what scents (may) spell out and ways to read it. Flavour 2014 3(Suppl 1):P11.

\section{School of Biosciences, Cardiff University, The Sir Martin Evans Building,} Museum Avenue, Cardiff CF10 3AX, UK 